\title{
Interplay of Rayleigh and Peierls Instabilities in Metallic Nanowires
}

\author{
D. F. Urban and Hermann Grabert \\ Physikalisches Institut, Albert-Ludwigs-Universität, D-79104 Freiburg, Germany
}

(Dated: November 20, 2018)

\begin{abstract}
A quantum-mechanical stability analysis of metallic nanowires within the free-electron model is presented. The stability is determined by an interplay of electron-shell effects, the Rayleigh instability due to surface tension, and the Peierls instability. Although the latter effect limits the maximum length also for wires with "magic radii", it is found that nanowires in the micrometer range can be stable at room temperature.

PACS numbers: 47.20.Dr, 61.46.+w, 68.65.La, 71.30.+h
\end{abstract}

Metal nanowires represent the ultimate limit of conductors down to a single atom in thickness. In recent years, experimental research on metal nanowires has burgeoned and various techniques have been employed to produce them for many different metals $[1,2,3,4,5]$. Remarkably, these nanowires are quite stable, although one might expect them to break up because of surface tension. Such an instability arises from classical continuum mechanics and is know as the Rayleigh instability [6]. Experimentally the stability was found to depend sensitively on the geometry of the wire. Yanson et al. $[2,3]$ have measured conductance histograms for alkali metal nanowires. At low temperature only wires with certain "magic radii" were stable. In another notable experiment [4, 5], Kondo et al. have produced long gold nanowires that were almost perfectly cylindrical in shape. Electron microscope images show lengths between 3 and $15 \mathrm{~nm}$ and diameters from 0.6 to $1.5 \mathrm{~nm}$. A histogram was built which again reflects stability for certain diameters only.

Many features of metallic nanowires can be explained in terms of a model of free electrons confined to the geometry of the wire [7]. At least for alkali metals, experiments on conductance quantization and current noise are in good agreement with theoretical predictions based on the Jellium model $[8,9,10,11,12]$. Furthermore, Kassubek et al. [13] have shown that a semi-classical calculation of the density of states of nanowires leads to stable magic radii, like those observed in experiments $[2,3]$. However, a puzzling conclusion of the semiclassical theory is that nanowires with a magic radius remain stable whatever their length.

There is substantial interest in the stability of nanowires, since they may serve as conductors in future nanocircuits. In particular, one would like to know whether they remain stable up to a length useful for applications. We therefore reexamine this problem using a fully quantum-mechanical analysis. It turns out that the suppression of the Rayleigh instability by electron shell effects, which is captured by the semiclassical theory, is now supplemented by an interplay between the Rayleigh and a novel Peierls-type instability missed in previous work. In fact, this latter quantum mechanical instability limits the maximal length of stable nanowires but still allows for wires in the micrometer range sufficient for most applications.

We treat the metallic nanowire as an open system connected to macroscopic metallic electrodes at each end. It is most naturally described within a scattering matrix approach [9]. The relevant thermodynamic potential is the grand canonical potential $\Omega$ which is related to the density of states $D(E)$ by

$$
\Omega=-k_{B} T \int d E D(E) \ln \left[1+e^{-\frac{(E-\mu)}{k_{\mathrm{B}} T}}\right],
$$

where $k_{B}$ is the Boltzmann constant, $T$ is the temperature and $\mu$ is the chemical potential specified by the macroscopic electrodes. The density of states can be calculated from the scattering matrix $S(E)$ by

$$
D(E)=\frac{1}{2 \pi i} \operatorname{Tr}\left\{S^{\dagger}(E) \frac{\partial S}{\partial E}-\frac{\partial S^{\dagger}}{\partial E} S(E)\right\} .
$$

A factor of 2 for spin degeneracy has been included. In order to examine the stability of a nanowire, we determine the energy change $\delta \Omega$ caused by a small deformation of the wire geometry. A nanowire is only stable if all small deformations result in an increase of $\Omega$.

The Jellium model considers free electrons that are kept in the wire by a confining potential chosen as a hardwall boundary. The corresponding Schrödinger equation can be solved by a separation ansatz

$$
\Psi(r, \varphi, z)=\sum_{\mu} C_{\mu}(z) \chi_{\mu}(r, \varphi ; z),
$$

in cylindrical coordinates $r, \varphi$ and $z$. This is motivated by the fact, that at a given position $z$ along the wire we can find a complete set of orthogonal transverse eigenfunctions $\chi_{\mu}(r, \varphi ; z)$ with eigenenergies $E_{\mu}(z)$.

Inserting this ansatz into the Schrödinger equation, we obtain a system of coupled differential equations for the coefficient functions $C_{\mu}(z)[14]$

$$
\begin{aligned}
& C_{\mu}^{\prime \prime}(z)+\left[E-E_{\mu}(z)\right] C_{\mu}(z)= \\
& -\sum_{\nu}\left\{2 A_{\mu \nu}(z) C_{\nu}^{\prime}(z)+\left[A_{\mu \nu}^{\prime}(z)+A_{\mu \nu}^{2}(z)\right] C_{\nu}(z)\right\},
\end{aligned}
$$


where a prime indicates differentiation with respect to $z$. For convenience, we use units with $\hbar^{2} / 2 m_{e}=1$, where $m_{e}$ is the electron mass. The coupling matrix reads

$$
A_{\mu \nu}(z)=\int_{0}^{2 \pi} d \varphi \int_{0}^{R(z, \varphi)} r d r \chi_{\mu}^{*}(r, \varphi ; z) \frac{\partial \chi_{\nu}(r, \varphi ; z)}{\partial z}
$$

In the following we want to examine a cylindrical wire and its sole classically unstable deformation - an axisymmetric one. In this case the geometry can be characterized by the radius function $R(z)$ and the coupling matrix can be written as $A_{\mu \nu}(z)=\mathcal{A}_{\mu \nu} \cdot R^{\prime}(z) / R(z)$, where $\mathcal{A}_{\mu \nu}$ is now independent of $z$. Eq. (4) simplifies to read

$$
\begin{array}{r}
C_{\mu}^{\prime \prime}+\left[E-E_{\mu}(R)\right] C_{\mu}=\sum_{\nu}\left[2 \frac{R^{\prime}}{R} \mathcal{A}_{\mu \nu} C_{\nu}^{\prime}+\right. \\
\left.\left\{\frac{R^{\prime \prime}}{R} \mathcal{A}_{\mu \nu}-\left(\frac{R^{\prime}}{R}\right)^{2}\left(\mathcal{A}_{\mu \nu}+\mathcal{A}_{\mu \nu}^{2}\right)\right\} C_{\nu}\right] .
\end{array}
$$

In the adiabatic approximation, which is justified if the radius varies slowly with $z$, the right hand side of Eq. (6) is neglected and the functions $C_{\mu}(z)$ can be determined from a WKB approximation [9]. This procedure is not valid for small wavelength modulations of the wire geometry that will be shown to limit the maximal length. Hence, we have to solve Eq. (6) systematically.

To determine the stability of a cylindrical wire with given radius $R_{0}$ and length $L$, we study small perturbations of its geometry given by

$$
R(z)=R_{0}\left(1+\varepsilon \sum_{q} b_{q} e^{i q z}\right) .
$$

The Fourier coefficients $b_{q}$ are chosen such that the total volume of the wire is unchanged by the deformation. Other physically reasonable constraints [15] are possible, but lead to similar results. We calculate the scattering matrix of the deformed nanowire connected to two cylindrical leads by matching solutions of the Schrödinger equation (6) at the boundaries. The density of states (2) and the grand canonical potential (1) are then derived as an expansion in the deformation parameter $\varepsilon$.

The grand canonical potential $\Omega$ is found to read

$$
\Omega(R(z))=\Omega^{(0)}\left(R_{0}\right)+\varepsilon^{2} \Omega^{(2)}\left(R_{0}\right)+\mathcal{O}\left(\varepsilon^{3}\right) .
$$

The leading order change $\Omega^{(2)}$ due to the deformation is of second order in the Fourier coefficients $b_{q}$ and can be written as

$$
\frac{\Omega^{(2)}}{L}=\sum_{q}\left|b_{q}\right|^{2} \alpha\left(q, R_{0}, L, T\right)+\mathcal{O}\left(\frac{1}{L}\right) .
$$

where the terms of order $1 / L$ include nondiagonal contributions which can be neglected if the wire is long enough. Hence the function $\alpha\left(q, R_{0}, L, T\right)$ essentially determines (a)

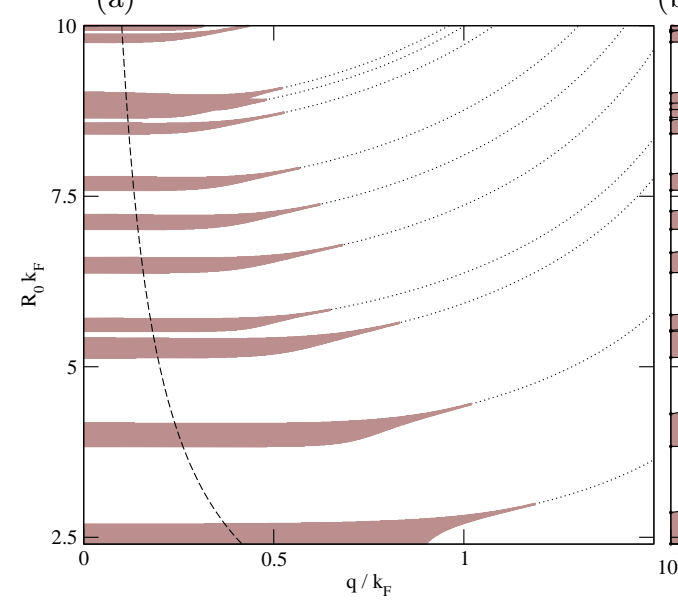

(b)

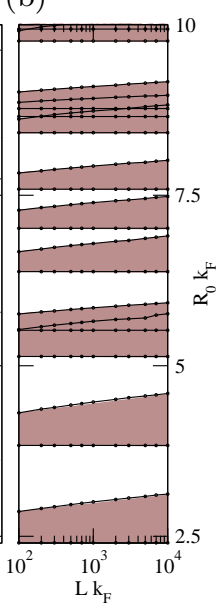

FIG. 1: a) Stability diagram for a cylindrical wire of length $L k_{F}=1000$ at zero temperature. Shaded areas are unstable. b) unstable radii as a function of the wire length

the stability of the nanowire and is therefore called stability coefficient. If $\alpha$ is positive for any value of the perturbation wave vector $q$, all small deformations of the cylindrical geometry lead to an increase of $\Omega$ and are therefore suppressed.

First we want to discuss the limit of zero temperature. Fig. 1(a) shows the stability diagram for a wire of length $L k_{F}=1000$ as a function of radius $R_{0}$ and perturbation wave vector $q$ at $T=0$, where $k_{F}$ is the Fermi wave vector. Shaded areas indicate a negative value of $\alpha$ and therefore instability. For comparison, the dashed line shows the stability criterion for the Rayleigh instability $\left(q R_{0}=1\right)$, and the dotted lines show the criterion of the Peierls instability

$$
q=2 k_{F}^{(\nu)}=2 \sqrt{E_{F}-E_{\nu}\left(R_{0}\right)},
$$

where $k_{F}^{(\nu)}$ is the Fermi wave vector of band $\nu$.

A sequence of stable and unstable radii can be extracted from such a stability diagram. This reflects the existence of so called magic stable radii, like they have been observed in experiments. Fig. 1(b) shows the extent of the regions of instability as a function of wire length, discussed below.

In order to understand this result it is useful to distinguish two contributions to $\alpha$ (Fig. 2):

$$
\alpha=\alpha^{(\mathrm{ch})}\left(q, R_{0}, L\right)+\alpha^{\text {(surf) }}\left(q, R_{0}\right) .
$$

The first term describes quantum effects due to the channel structure of electronic transport and the second one includes effects of surface tension and curvature energy.

For a given wire radius, $\alpha^{(\mathrm{ch})}$ as a function of $q$ shows a series of distinct negative minima. With increasing wire length, these minima get sharper and deeper, resulting in 


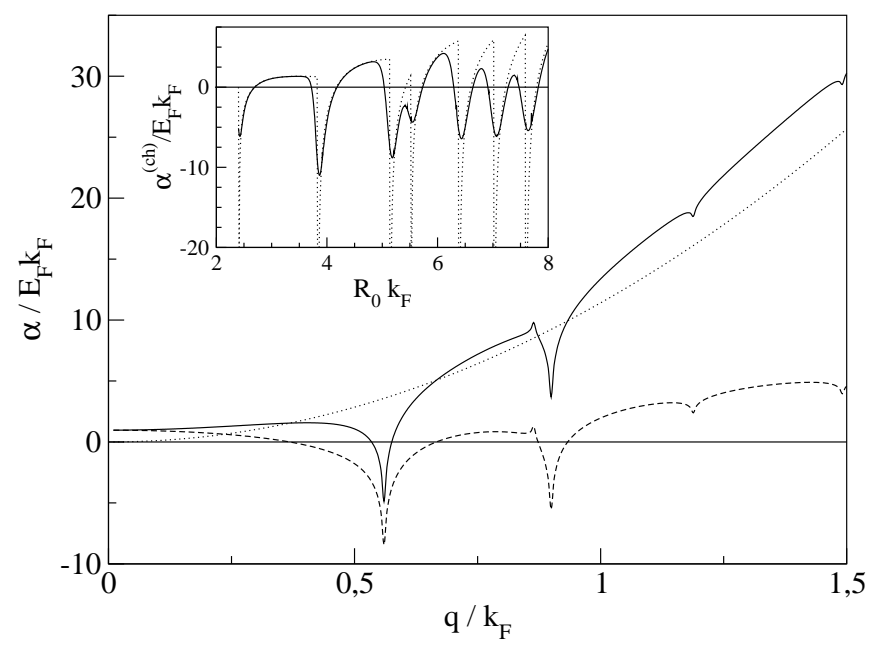

FIG. 2: The stability coefficients $\alpha$ (solid line), $\alpha^{(\mathrm{ch})}$ (dashed line) and $\alpha^{\text {(surf) }}$ (dotted line) as a function of $q$ for $R_{0} k_{F}=$ 5.75 and $L k_{F}=1000$. Inset: $\alpha^{(\mathrm{ch})}$ as a function of radius at $q=0.1 k_{F}$ and temperature $T=0$ (dotted line) and $T=$ $0.02 T_{F}$ (solid line), $T_{F}$ being the Fermi temperature.

a divergence for an infinitely long wire. These instabilities arise from an effect similar to the Peierls instability [16]. Nanowires are not purely one dimensional, but due to quantization perpendicular to the wire axis, electron transport is divided into distinct channels. Each channel has a quadratic dispersion relation and starts to contribute at a certain threshold energy, i.e., the eigenenergy $E_{\nu}$ of the corresponding transverse mode. This results in a sequence of quasi one dimensional systems with Fermi wave vectors $k_{F}^{(\nu)}$, as defined in Eq. (10). A perturbation of the translational symmetry of the system results in the opening of energy gaps in the electronic band structure. If a gap opens at the Fermi surface, the total energy of the system is lowered.

The detailed calculation here is different from the standard theory of the Peierls instability of a perturbed linear chain with periodic boundary conditions [16]. There, the energy change increases linearly with the length $L$ and $\alpha$ therefore is independent of $L$. Since the nanowire is part of a much larger system including the leads, the longitudinal wave vectors $k^{(\nu)}$ in the subbands are not restricted to multiples of $\frac{2 \pi}{L}$, like it is the case for an isolated system. Instead, the entire system formed by the leads and the wire determines the boundary conditions for the $k^{(\nu)}$. Hence, an analogous Peierls problem arises if we consider a linear chain of length $L_{0}$ that is perturbed by a potential $\sim V \cos \left(2 k_{F} z\right)$ acting within an interval of length $L$ only, where $L \ll L_{0}$. In this case, the change of the eigenenergies of states near $\pm k_{F}$ can be calculated by perturbation theory and the resulting energy dispersion relation is sketched in Fig. 3. The dispersion relation is smooth. Only in the limit $L \rightarrow \infty$ we recover the well known Peierls results with a jump at $k_{F}^{(\nu)}$.

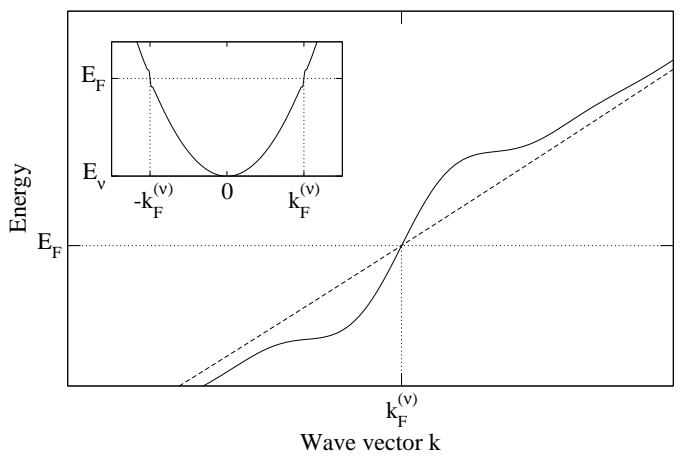

FIG. 3: Dispersion relation of a channel $\nu$ (solid line) due to a perturbation of the wire with a wave vector $q=2 k_{F}^{(\nu)}$. The inset shows the whole curve, while the main figure enlarges the vicinity of $k=k_{F}^{(\nu)}$. The dashed line represents the unperturbed parabolic dispersion.

In the case of a single conducting channel, labeled $\nu$, $\alpha^{(\mathrm{ch})}$ is given by

$$
\alpha^{(\mathrm{ch})}=\frac{16}{\pi} E_{\nu} k_{F}^{(\nu)}-\frac{4 E_{\nu}^{2}}{\pi q} \int_{\left|2 k_{F}^{(\nu)}-q\right| L}^{\left(2 k_{F}^{(\nu)}+q\right) L} \frac{x-\sin (x)}{x^{2}} d x,
$$

and has a minimum for $q=2 k_{F}^{(\nu)}$. For several open channels, $\alpha^{(\mathrm{ch})}$ includes terms analogous to Eq. (12) for each channel, as well as similar but more complicated terms that take into account coupling between any two channels having the same azimuthal symmetry. This leads to additional minima for $q=k_{F}^{(\mu)}+k_{F}^{(\nu)}$, that are less pronounced than those for $q=2 k_{F}^{(\nu)}$. Away from the Peierls-type instabilities, $\alpha^{(\mathrm{ch})}$ is positive and the nanowire is stabilized by electron shell effects.

The second term in Eq. (11) also tends to stabilize the wire. It is given by

$$
\alpha^{\text {(surf) }}=-\frac{2}{\pi} \sum_{\nu} k_{F}^{(\nu)}\left[\left(\mathcal{A}^{2}\right)_{\nu \nu}+\sum_{\mu}\left|\mathcal{A}_{\mu \nu}\right|^{2}\right] q^{2},
$$

with sums running over all open channels. A proportionality to $q^{2}$ is expected for the change of the surface and curvature energy of the wire due to a deformation with wave vector $q>0$. Using the explicit expressions for the coupling matrix elements one can show that $\alpha^{\text {(surf) }}(q)$ is always positive. Note that the negative surface contribution at $q=0$ that leads to the well-known Rayleigh instability is contained in $\alpha^{(\mathrm{ch})}$.

Now a coherent description of the effects determining the stability of a nanowire arises. Surface tension and curvature energy tend to stabilize the nanowire for deformations of small wavelength since the cost in energy increases $\propto q^{2}$. On the other hand, the classically expected Rayleigh instability for long wavelength perturbations is suppressed by electron shell effects that depend strongly on the wire radius. The channel structure of the 
(a)

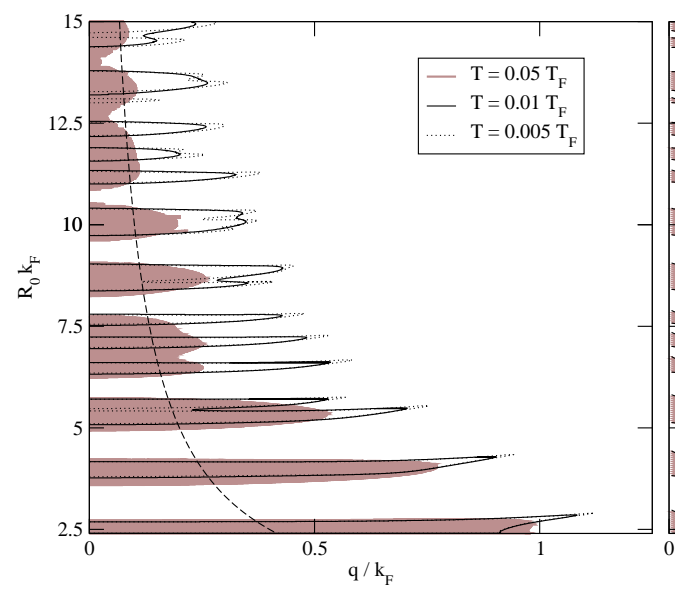

(b)

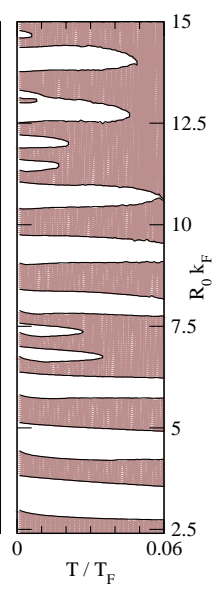

FIG. 4: (a) Stability diagram for a cylindrical wire of length $L k_{F}=1000$ at three different temperatures. The shaded area indicates instability at $T=0.05 T_{F}$, the solid and the dotted lines show the contours of the unstable regions for $T=$ $0.01 T_{F}$ and $T=0.005 T_{F}$ respectively, $T_{F}$ being the Fermi temperature. (b) unstable radii as a function of temperature

electronic transport results in several Peierls-type instabilities for perturbations of wave vector $q=2 k_{F}^{(\nu)}$ that become more pronounced with increasing wire length. The competition of these instabilities with the stabilizing effects of surface tension and curvature energy limits the maximal length of stable nanowires (Fig. 1). Nevertheless, stable wire geometries persist up to lengths greater than $L k_{F}=10^{4}$ (i.e. $L \sim 1 \mu m$ for $\mathrm{Na}$ and $\mathrm{Au}$ ) which is encouraging for the technological prospects of nanowires.

Now we turn to the effect of temperature. Increasing temperature results in a broadening and a flattening of the minima describing the Peierls instabilities (Inset Fig. 2). The stability diagram of a cylindrical wire of length $L k_{F}=1000$ is shown in Fig. 4(a) for three different temperatures. The Peierls instabilities disappear at higher temperature and the stability diagram approaches that of the classical Rayleigh instability, which is shown by the dashed line. Nevertheless islands of stability persist up to very high temperatures. Fig. 4(b) shows the extent of the unstable regions as a function of temperature and the increase of unstable configurations with growing temperature can be seen. The variation in the maximum temperature up to which the wires remain stable reflects the supershell structure observed in Ref. [3].

In our calculations the free electrons are kept in the wire by an infinitely high potential well ("hard-wall"). Since the electrons in a real metal are not so strongly confined, a more physical boundary condition would be a potential well of finite height depending on the electron work function. The main influence of this "soft-wall" boundary condition is to increase the effective radius of the cylindrical wire relative to the "hard-wall" case [17]. This has to be kept in mind when comparing our predic- tions to experiments.

Recently experiments found evidence of nanowires made out of dimerized chains of gold atoms [18]. This is not in contrast to our results, as in this experiment surrounding molecular hydrogen had a stabilizing effect on the wire. Also the Jellium model used for our calculations is at its limit of validity for a chain of atoms.

In conclusion, we have presented a fully quantum mechanical stability analysis of cylindrical nanowires, using the Jellium model. The stability of a nanowire is determined by the interplay of surface tension, shell effects and the Peierls instability. Our calculated stability diagrams prove the existence of certain stable magic radii like they have been found in experiments and give an explanation for the observed cylindrical geometry of these nanowires.

We thank C. A. Stafford, F. Kassubek and J. Bürki for valuable discussions. This research has been supported by the DFG through SFB 276 and the EU Network DIENOW.

[1] N. Agraït, J. G. Rodrigo, and S. Vieira, Phys. Rev. B 47, 12345 (1993).

[2] A. I. Yanson, I. K. Yanson, and J. M. van Ruitenbeek, Nature 400, 144 (1999).

[3] A. I. Yanson, I. K. Yanson, and J. M. van Ruitenbeek, Phys. Rev. Lett. 84, 5832 (2000).

[4] Y. Kondo and K. Takayanagi, Phys. Rev. Lett. 79, 3455 (1997).

[5] Y. Kondo and K. Takayanagi, Science 289, 606 (2000).

[6] S. Chandrasekhar, Hydrodynamic and Hydromagnetic Stability (Dover, New York, 1981).

[7] Some features of gold nanowires, in particular the helical structures observed in Ref. [5], require explicit inclusion of atomic structure and $5 \mathrm{~d}$ electrons [see e.g. E. Tossati et al., Science 291, 288 (2001)].

[8] J. A. Torres, J. I. Pascual, and J. J. Sáenz, Phys. Rev. B 49, 16581 (1993).

[9] C. A. Stafford, D. Baeriswyl, and J. Bürki, Phys. Rev. Lett. 79, 2863 (1997).

[10] F. Kassubek, C. A. Stafford, and H. Grabert, Phys. Rev. B 59, 7560 (1999).

[11] J. Bürki, C. A. Stafford, X. Zotos, and D. Baeriswyl, Phys. Rev. B 60, 5000 (1999).

[12] J. Bürki and C. A. Stafford, Phys. Rev. Lett. 83, 3342 (1999).

[13] F. Kassubek, C. A. Stafford, H. Grabert, and R. E. Goldstein, Nonlinearity 14, 167 (2001).

[14] C. Ulreich and W. Zwerger, Europhys. Lett. 41, 117 (1998).

[15] C. A. Stafford, F. Kassubek, J. Bürki, and H. Grabert, Phys. Rev. Lett. 83, 4836 (1999).

[16] R. E. Peierls, Quantum Theory of Solids (Oxford University Press, London, 1956).

[17] A. García-Martín, J. A. Torres, and J. J. Sáenz, Phys. Rev. B 54, 13448 (1996).

[18] S. Csonka, A. Halbritter, M. Mihaly, E. Jurdik, O. I. Shklyarevskii, S. Speller, and H. van Kempen, Phys. Rev. Lett. 90, 116803 (2003). 